COMECHINGONIA. REVISTA DE ARQUEOLOGÍA Número 16, primer semestre 2012, pp. 13-29, Córdoba

ISSN 0326-7911

\title{
APUNTES PARA UNA SEMIÓTICA DE LA MATERIALIDAD
}

\author{
José María Vaquer \\ ${ }^{1}$ CONICET Instituto de Arqueología, F.F.yL., U.B.A. 25 de Mayo 217 3er Piso - CP (1002 ABE). \\ E-mail:jmvaquer@yahoo.com
}

Presentado el: 05/12/2011 - Aceptado 20/03/2012

\section{Resumen}

En este trabajo propongo una reflexión teórico - metodológica sobre la aplicación de la Semiótica como marco para interpretar la materialidad. Para ello, considero necesario complementarla con otros dos enfoques: la Teoría de la Práctica y la Arqueología Conductual. Peirce considera al signo una entidad tripartita y dinámica, lo cual permite una serie de potencialidades para interpretar la cultura material. Considero la materialidad como una relación entre los sujetos y los objetos, a partir de un proceso histórico y contingente de objetivación que define los límites de cada uno. La Teoría de la Práctica focaliza en las prácticas sociales de los agentes como medio de producción y reproducción de la sociedad. La relación entre los agentes y la cultura material puede ser entendida a partir de las características de performance de los objetos, que los habilitan para participar en un cierto número de actividades. Por lo tanto, la Arqueología Conductual puede proveer el marco metodológico para interpretar la significación de la cultura material. Finalmente, considero la potencialidad de este marco para interpretar la relación entre el pasado y el presente a través de los significados atribuidos a la cultura material por las poblaciones actuales y los investigadores.

Palabras claves: Materialidad, Semiótica, Teoría de la Práctica, performance.

\begin{abstract}
In this paper I suggest some theoretical - methodological thoughts on the use of Semiotics as a frame to interpret materiality. To achieve this aim, it's necessary to complement Semiotic with Practice Theory and with the concept of performance as defined by Behavioral Archaeology. Peirce considered the sign as a tree - fold dynamic entity, which allow a potential to interpret material culture. I consider materiality as a relationship between subjects and objects, which from an historical and contingent process of objectivation defines them both. Practice Theory focus on the social practices of agents as a means of production and reproduction of society. The relationship between agents and material culture can be understood through the performance characteristic of objects, which enable them to participate in various activities. Therefore, Behavioral Archaeology can provide the methodological frame to interpret the meaning of material culture. Finally, I consider the potential of the theoretical frame presented to interpret the relationship between past and present, though meanings attributed to material culture by investigators and local populations.
\end{abstract}

Keywords: Materiality, Semiotics, Theory of Practice, performance.. 


\section{Introducción}

Uno de los problemas con los que se enfrentó la Arqueología desde su nacimiento como ciencia fue la existencia o no de una teoría propia que permita interpretar a las sociedades del pasado. Algunos autores consideraron que tal teoría estaba conformada por una serie de principios que relacionaban la estática del registro arqueológico con la dinámica de las sociedades "vivientes" (Binford 1994, 1999), otros propusieron una serie de leyes entre las instancias de ocupación de los sitos (contexto sistémico) y la manera en que el arqueólogo los encuentra en el presente (contexto arqueológico) (LaMotta y Schiffer 2001). Más allá de los ejemplos particulares, cada posición teórica utilizó diferentes disciplinas como marco interpretativo de mayor rango.

Con el nacimiento de la Arqueología Interpretativa o Postprocesual (prefiero este último término ya que considero que toda arqueología es interpretativa), se produjo un giro teórico y se comenzaron a buscar nuevas fuentes interpretativas para las sociedades del pasado. Dentro de ellas, tomaron preeminencia aquellas basadas en una combinación de Hermeneútica y Teoría Social, buscando distinguir los principios simbólicos que estructuraron a la cultura material del pasado (Hodder 1990; Parker Pearson y Richards 1994). Un paso más allá lo dieron los autores que incorporaron la Fenomenología como una herramienta metodológica, considerando la experiencia de los agentes sociales del pasado y de los investigadores en las interpretaciones (Tilley 1994; Thomas 1996). No obstante, para las explicaciones sistémicas de las sociedades del pasado, no existe un corpus teórico unificado en Arqueología.

Algunos investigadores demostraron su desacuerdo con esta situación, proponiendo diversas teorías para llenar el vacío teórico de alto rango (Leonard 2001; Renfrew 1994). Considero que la falta de una teoría unificada es una de las condiciones para que la Arqueología sea una ciencia creativa y que estimule la adecuación de diversos marcos teóricos para darle el giro que el investigador prefiera (Barrett 1994; Hodder 2001, 2007; Shanks y Tilley 1992). En mi caso particular, considero que la teoría que mejor se ajusta a mi visión de la Arqueología es la Teoría Social, por una serie de razones que voy a hacer explícitas a medida que desarrolle el trabajo.

Teniendo en cuenta los argumentos anteriores, entiéndase que este trabajo no es un intento de proponer "la" teoría de alto rango para la Arqueología, sino presentar una de ellas que resulta coherente y consistente, y que además es posible de aplicar a la cultura material. Parto de la premisa que la Arqueología es una ciencia interpretativa, lo cual implica que la cultura material no tiene un significado autoevidente, sino que es el trabajo de uno o varios sujetos interpretarlos, y que la explicación en Arqueología consiste en darle un sentido a los datos en lugar de testear hipótesis (Shanks y Hodder 1995; Thomas 2005). Esto también supone que la interpretación es un proceso que se realiza en el presente, por lo que dicho proceso debe tener una aplicación en el presente, es decir, considerar las consecuencias prácticas de los significados que interpretamos en la cultura material para todos los actores sociales involucrados. Por ello considero a la Arqueología como una ciencia social, ya que su objetivo debe construir narrativas del pasado como una praxis social, utilizando para ello una postura crítica (Funari et al. 2005).

Considerando los puntos mencionados hasta ahora, en este trabajo propongo un enfoque basado en tres herramientas teórico - metodológicas que se sitúan en tres niveles interpretativos: primero, la Teoría Social y particularmente la Teoría de la Práctica (Bourdieu 1977, 
1990; Giddens 1998, 2001) como el marco interpretativo de mayor rango. Este permite abordar preguntas generales, como por ejemplo ¿a través de qué mecanismos cambian o persisten las sociedades? O ¿cuál es el papel de los agentes sociales en el cambio?

Un segundo nivel lo constituye la Semiótica de Peirce (Short 2007) sumada a los estudios sobre la Materialidad (Meskell 2004; Miller 2005; Tilley 2006) y consiste en interpretar las relaciones entre los sujetos y los objetos desde una perspectiva semiótica que asigna una cierta responsabilidad a la cultura material en la producción y reproducción de los sistemas sociales. Este enfoque semiótico ha sido utilizado ya por varios investigadores (Lazzari 2005; Nielsen 2007a, 2007b; Preucel 2006; Salatino 2008; entre otros). Esto permite especificar aún más las preguntas correspondientes al primer nivel, por ejemplo ¿cuál es la relación entre los agentes sociales y la cultura material? Y ¿de qué manera los objetos, en tanto que conforman sistemas semióticos, tienen injerencia en la producción y reproducción social?

Finalmente, el tercer nivel se compone de modelos arqueológicos que permiten operacionalizar variables relacionadas con la interacción entre los sujetos y los objetos. Me refiero en particular a la Arqueología Conductual (LaMotta y Schiffer 2001; Schiffer 1999), que, dentro de su programa, incluye el concepto de performance como las cualidades de un objeto que lo habilitan para participar de ciertas relaciones sociales (Nielsen 1995). Este tercer nivel permite abordar la cultura material en términos de preguntas como ¿cuáles son las características de performance de un objeto que alientan o desalientan ciertas prácticas sociales? Y ¿las características de performance de los objetos se relacionan con las posibilidades perceptivas de los mismos, y por lo tanto, con la inclusión en sistemas semióticos particulares?

Estos tres niveles con sus respectivas preguntas constituyen el nudo del trabajo. Queda un nivel más, pero considero que implica un plano diferente de discusión y merece ser tratado como un tema aparte. Me refiero a la aplicabilidad de este marco para interpretar las relaciones entre los agentes sociales del presente con la cultura material. Dentro de la categoría "agentes sociales" incluyo a todas las personas que tienen una relación con la cultura material, como los investigadores, las poblaciones locales, las autoridades que trabajan con el patrimonio e incluso los eventuales turistas. Desde una perspectiva informada por los tres niveles de análisis propuestos, el abanico de posibilidades interpretativas se ve potenciado, ya que uno de los postulados que voy a desarrollar más adelante es que el proceso de significación de la cultura material no termina nunca. Esta no es una idea nueva, ya que varios autores resaltaron este punto (Funari et al 2005; Politis y Pérez Gollán 2007; Shanks y Tilley 1992; entre otros). Lo importante del marco es que, al entender las relaciones entre la cultura material y los agentes del pasado y del presente en los mismos términos, permite que las interpretaciones construidas en cualquiera de los ámbitos sean aplicables al otro. En este sentido, es un marco solidario entre el pasado y el presente, y esto produce una responsabilidad de los investigadores sobre las consecuencias prácticas de sus discursos sobre el pasado y el presente.

Vamos a comenzar desarrollando los tres niveles de análisis, y en último término considero las relaciones entre el pasado y el presente. Es necesario mencionar que en este trabajo, por cuestiones de espacio y de objetivos, no voy a desarrollar la aplicación del marco a un ejemplo arqueológico. Para ello, invito al lector a consultar Vaquer (2010a, 2010b, 2011a) y Vaquer et al. (2010). 


\section{Desarrollo}

Primer Nivel: La Teoría Social y la Teoría de la Práctica

En esta sección desarrollo algunos conceptos sobre la Teoría Social en general, y la Teoría Social Posmoderna en particular. Me enfoco en esta última porque es la más solidaria con el marco teórico propuesto, y comparto las críticas que realiza a la Teoría Social de corte objetivista (para una discusión más amplia al respecto, ver Giddens 2001 y Ritzer y Smart 2001). Finalizo la sección introduciendo el problema de la relación entre lo macro y lo micro en la Teoría Social y presentando una síntesis de la Teoría de la Práctica de Bourdieu (1977, 1990) y la Teoría de la Estructuración de Giddens (1998).

La Teoría Social está lejos de ser un campo unificado y claramente delimitado. De acuerdo con Ritzer y Smart (2001) recibió aportes de diversas disciplinas como la sociología, la economía política, la filosofía y los estudios culturales. Lo que unifica a este campo es la existencia de un conjunto sustancial de autores definidos como "clásicos" a los que se retorna permanentemente (principalmente Marx, Weber y Parsons); y que su principal objetivo es interpretar el "fenómeno social". El objetivo explícito de la Teoría Social es dar cuenta de la manera en que el mundo social cambia, y las formas y estilos requeridos para generar explicaciones efectivas de las condiciones sociales transformadas en las que vivimos (Ritzer y Smart 2001).

Una de las características del pensamiento social es una constante búsqueda de una síntesis o teoría, o de un enfoque que ordene y explique apropiadamente "lo social". La constante proliferación de nuevos enfoques demuestra la imposibilidad de reducir la sociedad a un ordenamiento teórico fijo. Debido a esto, la noción de una acumulación progresiva del conocimiento sobre el mundo social representada por el desplazamiento de un paradigma por otro mejor equipado para explicar la realidad social, no se corresponde con una descripción adecuada del campo de la Teoría Social (Ritzer y Smart 2001). Una de las causas de este proceso es lo que Giddens (2001) denomina la "doble hermenéutica" de las ciencias sociales: la relación con los sujetos de estudio es compleja porque la producción de teoría social no se encuentra desconectada del mundo social que describe. Las ideas, conceptos y explicaciones se infiltran en los contextos que intentan explicar, modificando tanto al mundo social como a los científicos sociales.

Teorías Sociales Posmodernas

Según Crook (2001), el posmodernismo en Teoría Social se caracteriza por un rechazo de las grandes narrativas y los esquemas racionalistas unificadores que caracterizan a las posturas teóricas dominantes de corte objetivista. Las grandes narrativas son explicaciones de la historia social que muestran al cambio social como predecible o necesario. Los esquemas unificadores o totalizadores son aquellos que proponen alguna fuerza subyacente como la explicación de todos los fenómenos sociales (Ritzer y Goodman 2001). En vez de grandes narrativas, los postmodernos proponen un retorno a lo local, lo subordinado y lo irracional.

Otros autores adoptan posiciones más radicales. Para Ritzer y Goodman la posmodernidad es el reconocimiento de "la intratable contingencia de la modernidad" (2001: 151), es decir, que la situación actual es el producto de una serie de accidentes históricos y no de una 
fuerza esencial. Desde una óptica moderna, el éxito de las ciencias sociales es una transparencia incrementada del objeto social, es decir, el revelado de fuerzas y procesos subyacentes y esenciales. Para los postmodernos, el éxito se define por revelar la contingencia radical de la sociedad y la apertura de nuevas posiciones subjetivas y proyectos sociales locales.

El foco en la contingencia conlleva una serie de presupuestos epistemológicos (Ritzer y Goodman 2001). El primero de ellos es la descentralización, que involucra abandonar la idea de que existe un centro, un origen que determina fenómenos subsecuentes y periféricos. El segundo de ellos es la deconstrucción, basada en demostrar que los fenómenos que son presentados como marginales y derivados son siempre necesarios para sostener lo que se presenta como central y original.

Los postmodernos proponen que la relación entre la teoría y la sociedad también es contingente: los elementos de la teoría y la manera en que se expresan son parte de múltiples sistemas contradictorios. Son determinados por la biografía del teórico / a, el contexto social contemporáneo, la historia de la disciplina del investigador / a; y las contingencias de los accidentes históricos, biográficos, lingüísticos y sociales. En este sentido, los postmodernos consideran que la naturaleza esencial de la sociedad es la contingencia, y que el rol central de la teoría es revelarlo.

Agencia y estructura: la relación entre lo micro y lo macro

Barnes (2001) propone que todos los campos de investigación empírica enfrentan en alguna medida problemas entre lo macro y lo micro. Esto implica preguntarse cómo las propiedades y propensiones de los objetos "macro" se relacionan con los objetos "micro", y cómo los resultados obtenidos en un campo de estudio se relacionan con el otro. Para este autor, casi todas las explicaciones de las relaciones macro/micro involucran reduccionismos, dualismos o alguna forma de pragmatismo o constructivismo.

Por analogía con las demás ciencias, la explicación en las ciencias sociales consistió en unir las acciones las causas e influencias independientes de ellas mismas. La conducta de los agentes sociales fue explicada en función de un conjunto de normas, que a su vez referían a un orden mayor, la estructura social o el sistema social. La estructura social se compone, entonces, por reglas y normas ordenadas de acuerdo con un status para conformar instituciones que enmarcan (y explican) las acciones individuales. En este sentido, la estructura fue considerada como real, separada, anterior, macro y explicativa. Las acciones manifiestan patrones que pueden ser descriptos y considerados como una entidad macro social separada, que a su vez, explica las acciones (Barnes 2001).

Una de las principales críticas que recibieron los enfoques basados en la estructura es que consideran a los individuos humanos como productores pasivos de las acciones según las especificaciones de los modelos sociales y culturales de su momento. La capacidad de los seres humanos de elegir se denomina agencia y los autores la definen de acuerdo con el marco conceptual en el cual trabajan. Callinicos (2004: XIX) propone una "concepción ortodoxa del agente" en las ciencias sociales que implica la idea de que la acción de los agentes puede ser explicada intencionalmente al adscribir a los actores creencias y deseos que los hacen actuar de la manera en que lo hacen. Otro corolario de esta definición es que los agentes al actuar ejercen cierto poder. 
Por su parte, Giddens (1998) propone que ser un agente es ser capaz de desplegar un espectro de poderes causales, incluido el poder de influir sobre el desplegado por otros. Una acción nace de la aptitud del individuo para "producir una diferencia" en un estado de cosas o curso de sucesos preexistentes. Un agente deja de ser tal si pierde la aptitud de "producir una diferencia", o sea, de ejercer alguna clase de poder. En la óptica de este autor, el poder es concebido principalmente como aptitud transformadora, como un recurso que es una propiedad estructural del sistema social y los actores utilizan y reproducen en el curso de una interacción.

Uno de los problemas específicamente planteados por la Teoría de la Práctica es justamente la relación entre la agencia y la estructura. Los dos autores cuyas propuestas sintetizo a continuación tienen un enfoque similar: las prácticas son el nexo que une a la estructura y a la agencia en la reproducción social. La principal diferencia es que Giddens (1998) coloca mayor énfasis en la capacidad transformadora del agente social mientras que Bourdieu (1977) le da mayor relevancia a los aspectos estructurales a través del habitus.

La Teoría de la Práctica de Bourdieu

De acuerdo con Bourdieu (1977), para escapar del realismo de la estructura que convierte a las relaciones objetivas en totalidades constituidas fuera de la historia individual y grupal, es necesario pasar de las regularidades estadísticas o estructuras algebraicas (el opus operatum) al principio de producción del orden observado (modus operandi), es decir, a la teoría del modo de generación de las prácticas. Para ello, propone el concepto de habitus que define como:

“Un sistema subjetivo pero no individual de estructuras internalizadas, esquemas de percepción, concepción y acción comunes a todos los miembros de un grupo o clase, constituyendo de esta manera la precondición de toda objetivación. La coordinación objetiva de las prácticas y el compartir una cosmovisión pueden ser fundados en la perfecta impersonalidad e intercambiabilidad de prácticas y visiones singulares" (Bourdieu 1977: 86).

Este sistema de disposiciones tiende a reproducir las estructuras objetivas de las cuáles es el producto, ya que se encuentra determinado por las condiciones pasadas que lo produjeron. El habitus es la fuente de una serie de movimientos que son objetivamente organizados como estrategias por los agentes sociales. De esta manera, se manifiesta como un sistema socialmente constituido de estructuras cognitivas y motivadoras que se confronta con situaciones socialmente estructuradas en las cuales se definen los intereses de los agentes.

Uno de los efectos fundamentales de la orquestación del habitus es la producción de un mundo de sentido común dotado de la objetividad asegurada por el consenso en el significado de las prácticas y el mundo, la armonización de las experiencias de los agentes y el refuerzo continuo que cada uno recibe de la expresión, individual o colectiva, improvisada o programada, de experiencias similares o idénticas. En este sentido, las estimaciones prácticas dan un peso desproporcionado a las experiencias anteriores: las estructuras características de un determinado tipo de condiciones de existencia, a través las necesidades económicas y sociales producen las estructuras del habitus que se convierten a su vez las bases de la percepción y la apreciación de toda experiencia subsecuente. 
Para Bourdieu (2002), todas las sociedades se presentan como espacios sociales, es decir, estructuras de diferencias que son posibles de comprender si se elabora el principio generador que fundamenta las diferencias en la objetividad. Este principio es la estructura de la distribución de las formas de poder o especies de capital eficientes en el universo social considerado. El espacio social puede ser considerado como un campo, un campo de fuerzas cuya necesidad se impone a los agentes sociales que han entrado en él y como un campo de luchas en el cual los agentes se enfrentan con medios y fines diferenciados según su posición en la estructura del campo de fuerzas, contribuyendo de esta manera a conservar o transformar su estructura.

Giddens: la Dualidad de la Estructura y la Teoría de la Estructuración

Según Giddens (1998) el dominio primario de las ciencias sociales no es la vivencia del actor individual ni la existencia de alguna forma de totalidad societaria, sino las prácticas sociales ordenadas en un espacio y un tiempo. Para este autor las actividades sociales humanas se auto-reproducen, son recursivas. Esto implica que los actores no las crean, sino que las recrean a través de los mismos medios por lo que se expresan en tanto actores. En sus actividades los agentes reproducen las condiciones que hacen posibles esas actividades.

Los conceptos que componen el núcleo de la Teoría de la Estructuración son estructura, sistema y dualidad de la estructura. La estructura para Giddens es un "orden virtual de relaciones transformativas" (1998: 54). Esto implica que los sistemas sociales, en tanto prácticas sociales reproducidas, no tienen estructuras, sino que presentan propiedades estructurales. La estructura existe como presencia espacio temporal sólo en las actualizaciones de esas prácticas y como huellas mnémicas que orientan la conducta de los agentes humanos. Sistema es entendido como las relaciones reproducidas entre actores o colectividades organizadas como prácticas sociales regulares.

La dualidad de la estructura implica que las reglas y recursos que se aplican a la producción y reproducción de una acción social son, al mismo tiempo, los medios para la reproducción sistémica. La constitución de los agentes y las estructuras no son dos conjuntos de fenómenos dados independientemente, no forman un dualismo, sino que para Giddens representa una dualidad. Esto significa que las propiedades estructurales de los sistemas sociales son tanto un medio como un resultado de las prácticas que ellas organizan de manera recursiva. En la reproducción de las propiedades estructurales, los agentes también reproducen las condiciones que hacen posible esa acción.

\section{Segundo Nivel: Materialidad y Semiótica}

Materialidad y cultura material no significan lo mismo. De hecho, la diferencia entre ambos conceptos radica en la manera que definen las relaciones entre los sujetos y el mundo material. Aunque "cultura material" reconoce que la relación dialéctica entre los objetos y los sujetos, el énfasis se encuentra puesto en el sistema simbólico detrás de los objetos. El reclamo hacia esta postura es que el objeto es conceptualizado como un indicador de algo más, y se deja de lado al objeto en sí mismo (Olsen 2003). Además, al considerar al sistema simbólico detrás del objeto se reproducen divisiones entre el cuerpo, la mente y el mundo propias de la Modernidad al tratar a los objetos como compuestos por dos componentes diferentes: la materialidad del objeto y el sistema simbólico a ser interpretado (Jones 2007; ver también Thomas 1996). 
Por otro lado, Meskell (2004) reconoce dos tendencias dentro de los estudios de cultura material. Una de ellas, que denomina "la tendencia empírica" se focaliza en el análisis de los objetos en términos tecnológicos. La segunda "tendencia teórica" trata con las connotaciones interpretativas alrededor y más allá del objeto. Ambas fallan, para la autora, en no considerar la dialéctica entre las personas y los objetos. Esto implica reconocer, por un lado, que la relación entre los objetos y las personas es específica a cada momento cultural particular; y por el otro que los sujetos y los objetos se constituyen mutuamente. La forma que propone para lograr este objetivo es a través de la Fenomenología, al explorar las prácticas, la incorporación, la experiencia, la agencia, biografía, reflexividad y la narrativa en cada contexto. Sin embargo, para Meskell (2004) esto último no implica imbuir a los objetos de agencia y convertirlos en sujetos, sino definir los límites de la agencia dentro de cada contexto cultural.

Uno de los conceptos principales a la hora de abordar la materialidad, es el concepto de objetivación (Miller 2005; Tilley 2006). Miller (2005) lo define como un proceso en el tiempo por el cual el hecho de crear formas materiales crea consciencia o capacidad, y por lo tanto transforma a la forma y a la consciencia. Las nociones de humanidad que maneja una sociedad determinada no son previas al proceso de objetivación, sino que este mismo proceso produce lo que parecen sujetos autónomos y objetos autónomos. Por lo tanto, las nociones de "sujeto" y "objeto" son meras apariencias que emergen en el proceso de objetivación. El corolario de esta postura es que si las formas materiales tienen consecuencias para las personas de manera autónoma de la agencia humana, puede considerarse que los objetos poseen una agencia que produce dichos efectos. En este sentido, hay posturas que consideran que los objetos poseen agencia propia y no pueden diferenciarse de los sujetos, o que consideran que los objetos producen efectos a partir de la agencia distribuida de los sujetos que los crean. Un paso más adelante en esta dirección lo constituyen los enfoques denominados "simétricos", donde se le da un mismo status a los sujetos y a los objetos (González - Ruibal 2007; Latour 2005; Olsen 2003).

Al centrarse en la relación dialéctica entre los sujetos y los objetos, los enfoques basados en la materialidad superan las posturas dicotómicas y reconocen la importancia de las prácticas sociales como nexo entre ambos. También es importante que las relaciones entre los sujetos y los objetos son mutuamente constitutivas.

Ahora bien, ¿de qué manera podemos abordar metodológicamente la relación constitutiva entre los sujetos y los objetos? Teniendo en cuenta la propuesta de Meskell (2004) expuesta anteriormente, la solución no radica en atribuirle agencia a los objetos y punto. Es necesario considerar que el proceso de objetivación es contingente y particular a cada sociedad y momento histórico, al igual que la agencia. Los límites de la objetivación y de la agencia deben definirse de acuerdo con el contexto.

Keane $(2005,2007)$ propone que la semiótica de Peirce es adecuada para comprender la materialidad. Según este autor, para Peirce los signos se encuentran en un mundo material de consecuencias, donde las circunstancias concretas son esenciales para las posibilidades de significación. En este sentido, el modelo de signo que propone Peirce es procesual, ya que los signos dan nacimiento a nuevos signos en un proceso constante de significación que involucra sociabilidad, lucha, historicidad y contingencia. Por lo tanto, la relación entre las posibilidades de los signos, las interpretaciones y los objetos es compleja. 
Otra de las ventajas de la semiótica de Peirce es que considera al signo como una entidad triádica: el objeto, el signo y el intérprete. Dentro de este marco, el intérprete siempre es considerado como una respuesta, o un rango potencial de respuestas (Short 2007). Esto implica que la significación tiene un componente inherentemente práctico y activo. El intérprete también se encuentra dotado de una capacidad de acción.

Uno de los puntos fundamentales, y que se relaciona directamente con la materialidad, son las características del objeto como determinantes de la significación. Un signo, al estar compuesto por tres elementos, hace referencia no solamente al signo y al objeto que representa, sino a un intérprete potencial. Lo importante es la interpretabilidad, la interpretación potencial, que, a diferencia de la semiología de Saussure (1993) no es arbitraria, debe ser justificada por un propósito vinculado al objeto. Además, la significación se encuentra determinada por las características del objeto: el objeto determina al signo, que a su vez determina al intérprete en tanto respuesta potencial. La relación entre el objeto y el intérprete es una de propósito. Por lo tanto, en este marco la significación depende directamente de las características del objeto, y de las capacidades de respuesta del intérprete. La relación es material y práctica.

Keane (2007) propone el concepto de "ideología semiótica" como una expansión del concepto de "ideología del lenguaje" desarrollado por la Antropología Lingüística. La objetivación es una precondición de la conciencia reflexiva, lo cual implica que no es el fin de los procesos dinámicos de significación, sino un momento de los mismos. Las ideologías del lenguaje son una instancia especial dentro de un principio más general de reflexividad dentro del proceso de creación y transformación de los fenómenos sociales, donde la reflexividad peculiar del lenguaje tiene un papel principal en la regulación de los demás dominios semióticos. Sin embargo, un enfoque basado en la materialidad no debe tomar a los objetos como indicadores de "algo más" como el lenguaje, sino intentar contar historias a partir de los objetos mismos. Por lo tanto, hay que considerar la forma en que la cultura material trabaja independientemente, o en contradicción con, el ambiente discursivo que la rodea.

Una de las propuestas de Keane (2007) es que las ideologías semióticas superan a las ideologías del lenguaje porque la distinción entre lo que se considera lenguaje y lo que no, en una sociedad particular, se construye ideológicamente y difiere entre contextos sociales e históricos. De esta manera, las ideologías semióticas se centran en las relaciones entre las palabras y las cosas, teniendo en cuenta que las ideas y las prácticas que involucran no poseen solamente consecuencias lógicas, sino también efectos causales entre sí en un rango amplio de campos sociales aparentemente distintos. Para ello, introduce el concepto de "economía de la representación" (Keane 2007: 18) que implica las prácticas e ideologías asociadas en una relación dinámica y dialéctica. Esta "economía de la representación" sitúa a las palabras, las cosas y las personas (junto con otros seres con agencia) en el mundo, definiendo las propiedades de cada uno y su relación mutua. En un contexto social determinado se encuentran en juego múltiples "economías de representación", con sus diferentes elementos sujetos a diferentes lógicas y temporalidades causales. La ideología semiótica une y alinea las ofertas de las diferentes economías, involucrando supuestos básicos sobre qué tipos de seres habitan el mundo, cuáles cuentan como posibles agentes y cuáles son las precondiciones y las consecuencias de la acción moral.

En resumen, las ideologías semióticas son un reflejo y un intento de organizar la experiencia de los agentes sobre la materialidad de las formas semióticas. Cualquier elemento 
que sea incluido en una práctica semiótica funciona dentro la experiencia perceptible en virtud de sus propiedades materiales, por lo que las ideologías semióticas estabilizan los significados en formas repetibles que son la condición mínima para su reconocimiento, su circulación por el espacio social y su capacidad de extensión temporal. Por lo tanto, la habilidad de los agentes de reconocer estas formas como "lo mismo" y actuar en consecuencia depende de cómo se encuentran enmarcadas, ya que la materialidad siempre se encuentra abierta a otras posibilidades de significación. Este último punto es importante, ya que es el elemento de cambio presente en las ideologías semióticas.

Tercer Nivel: La Arqueología Conductual y la performance

La Arqueología Conductual (LaMotta y Schiffer 2001; Nielsen 1995; Schiffer 1999) se encuentra basada en el estudio de las interacciones entre las personas y los objetos materiales. La unidad de análisis para esta perspectiva es la conducta, que incluye tanto a las personas como a los objetos. En este sentido, se relaciona directamente con el proceso de objetivación descripto anteriormente. Sin embargo, es necesario hacer algunas aclaraciones. Adoptar el marco metodológico de la Arqueología Conductual no implica que acepte todas las implicancias teóricas. Por ejemplo, el objetivo de la Arqueología Conductual es "subsumir los fenómenos empíricos bajo proposiciones nomotéticas y las generalizaciones empíricas que especifican regularidades en procesos conductuales en varias escalas" (LaMotta y Schiffer 2001: 18). En este sentido, no considero la posibilidad de construir proposiciones nomotéticas ya que en mi visión laArqueología es una ciencia interpretativa. También rechazo la visión sistémica de este enfoque. Otra aclaración importante es respecto al concepto de "conducta". De acuerdo con Pauketat, la conducta se refiere a la actividad humana abstracta orientada a fines, mientras que la perspectiva que tomo en este trabajo busca entender las prácticas, definidas como "acciones y representaciones homólogas que varían entre contextos o incluso eventos si las formas rutinizadas tienden a permanecer iguales" (Pauketat 2001a: 86).

Como mencioné anteriormente, el primer punto importante es la relación entre las personas y los objetos. Los objetos poseen propiedades formales que son cruciales para la incorporación del objeto en actividades específicas. Según Schiffer (1999: 123) la performance es "la relación mínima entre un interactor con otro en una interacción discreta". Asociados a este concepto se encuentran las nociones de características de performance, referida a una capacidad o competencia ejercida por un interactor en una performance específica; y las características de performance sensoriales, que se refieren al uso de los sentidos. Por su parte, Nielsen (1995) define a la performance como las capacidades conductuales de los artefactos que son el resultado del diseño y les permiten crear y recrear formas específicas de interacción social.

Por lo tanto, la relación entre los sujetos y los objetos se encuentra mediada por las características de performance. Las mismas se refieren a los atributos formales de los objetos que les permiten formar parte de una serie de actividades y no otras (aunque las funciones nunca son excluyentes) en términos de un abanico de posibilidades. Un objeto, en virtud de su diseño, es apto para formar parte de una serie de actividades (por ejemplo una silla no puede ser utilizada como sombrero). Las características de performance tienen dos lados: el lado formal que explicité y un lado sensorial, aunque en la práctica es imposible distinguir entre los dos. Además de las propiedades formales que permiten la inclusión en actividades específicas, los objetos también tienen propiedades sensoriales que los hacen participar en diferentes modalidades sensoriales. Es en este sentido, a partir del análisis de las caracte- 
rísticas de performance de los objetos, que considero pertinente la noción de "agencia de los objetos". En virtud del diseño y de situaciones históricas contingentes los objetos nos "hacen cosas hacer cosas".

Aunque parezca incoherente, la teoría de la performance propuesta por Schiffer en sus últimos trabajos no difiere demasiado de las propuestas basadas en el estudio de la Materialidad discutidas anteriormente. El reclamo de los autores dentro de este marco es una "vuelta al objeto", dejando de lado la obsesión por identificar sistemas simbólicos. La Arqueología Conductual, desde una perspectiva social como la propuesta por Nielsen (1995) también intenta abordar las características intrínsecas a los objetos para determinar su posibilidad de participación en prácticas sociales. Como desarrollé en las secciones anteriores, una de las ventajas de la semiótica de Peirce es su énfasis en las características sensoriales de los objetos como motivadoras de la significación. Por lo tanto, la Arqueología Conductual, al centrarse en las características de performance de los objetos permite incluirlos en sistemas semióticos plausibles de ser analizados a partir de su materialidad.

Con respecto a la relación entre la Arqueología Conductual - de corte objetivista y por lo tanto criticada tanto por Giddens como por Bourdieu - y la Teoría de la Práctica, es necesario aclarar algunos puntos. Schiffer intenta subsumir los fenómenos dentro de leyes generales, y en este punto es donde las teorías entran en conflicto. Pero más allá de la pretensión de Schiffer, las herramientas metodológicas de la Arqueología Conductual pueden ser utilizadas dentro de un marco que enfatice las prácticas sociales. El objetivo no sería buscar leyes generales de conducta, sino que, a través de las características de performance de los objetos, interpretar sus posibles significados a través de su inserción en prácticas sociales. Y, como se comentó más arriba, este es un proceso semiótico.

Por lo tanto, la utilización de la Arqueología Conductual se encuentra restringida, dentro del marco adoptado en este trabajo, a su bagaje metodológico. Sé que no es posible dividir entre metodología y teoría, ya que una implica a la otra, pero en este caso, los resultados obtenidos dentro de la metodología de la Arqueología Conductual pueden ser interpretados dentro de los estudios semióticos de la materialidad, y dentro de la Teoría de la Práctica.

\section{Conclusiones}

Entonces, y para realizar una síntesis, propongo que la Arqueología, en tanto Ciencia Social, debe nutrirse y ser consciente de las discusiones de la Teoría Social. Esto no implica que considere que toda Arqueología debe ser una Arqueología Social, ya que estoy de acuerdo con Hodder (2001) que una pluralidad de enfoques es constructiva para nuestra disciplina.

Por otro lado, la categoría de "lo social" también resulta problemática (Latour 2005), por ello para evitar reificar a "lo social" como un reino elusivo o una categoría abstracta, propongo hacer una arqueología de las prácticas sociales. "Lo social", en estos términos, son las prácticas realizadas por agentes entendidos, que en sus interacciones producen y reproducen a la sociedad. De esta manera, se supera la dicotomía entre lo micro y lo macro, ya que las prácticas sociales pertenecen a ambos dominios, o mejor dicho, los estructura en forma recursiva. También supera la dicotomía entre los enfoques objetivistas y subjetivistas, a partir de reconocer la existencia del habitus como una estructura estructurante conformada a partir de condiciones objetivas que opera a nivel subjetivo como disposiciones. 
El enfoque que adopto es considerar que las prácticas de los agentes son constitutivas de la sociedad en términos de instituciones que superan la extensión temporal de las prácticas. En este sentido, el concepto de institución propuesto por Giddens (1998) es similar a la noción de campo de Bourdieu (2002). Cuando las instituciones se prolongan en el tiempo y en el espacio podemos considerar a las mismas como tradiciones (Barrett 1994; Pauketat 2001a, 2001b), incluso las tradiciones son constituidas por los arqueólogos investigando esas tradiciones.

Cuarto Nivel: Una Arqueología para el presente

Queda como pendiente definir el "cuarto nivel" de aplicación del marco, es decir, la manera en que esta propuesta resulta rentable para interpretar las relaciones entre la cultura material y los agentes sociales en el presente. Este nivel ocupa el último lugar dentro del esquema lógico del marco, ya que depende de los tres niveles anteriores para su definición. Pero, desde un punto de vista ético, ocupa el primer lugar.

La principal premisa del argumento es que la Arqueología es una ciencia del presente, que debe interpretar el pasado para actuar sobre el presente (Shanks y Tilley 1992). Tenemos una responsabilidad sobre las interpretaciones que generamos: ¿para quién narramos el pasado? Y aún más importante: ¿qué consecuencias se siguen de nuestras interpretaciones? Estas dos preguntas no deben hacerse una vez que la investigación ha concluido, sino que son el nudo a partir del cual armar todo nuestro esquema interpretativo. Cada investigador tendrá su propia postura al respecto, pero si consideramos a la Arqueología como un conjunto de prácticas, en coherencia con el marco presentado, esas prácticas son constitutivas de la sociedad.

$\mathrm{Al}$ unir los tres niveles presentados en este trabajo, nos encontramos con que la materialidad es una relación siempre actuante, que indefectiblemente nos relaciona con un "otro", que en este caso es el pasado. Esta relación depende de sistemas semióticos, en particular de varias ideologías semióticas que pretenden fijar ciertos significados. Pero una de las características más importantes del enfoque de Peirce sobre la semiótica es que el proceso de significación se encuentra abierto de manera constante, hecho también enfatizado por Keane. Siempre existe lugar para nuevos significados, y las ideologías semióticas nunca son exhaustivas ni finales. Por lo tanto, nuestro diálogo con el pasado nunca tiene fin.

Además de la otredad representada por el pasado, la Arqueología también dialoga con los demás agentes sociales relacionados con la cultura material, principalmente los pueblos originarios y comunidades para los que los vestigios del pasado son parte de sus vidas cotidianas. Y como tales, también son interpretados a su vez. La responsabilidad que tenemos con ellos es descentrar nuestro discurso sobre el pasado, y presentarlo como una interpretación más que no invalida los discursos locales y propios sobre el pasado. Esta es una de las propuestas que se desprende de la Teoría Social Posmoderna comentada anteriormente.

\section{Perspectivas Futuras}

En este trabajo presenté una propuesta para interpretar la cultura material a partir de un enfoque basado en la Teoría Social y la Semiótica de Peirce. Como mencioné al principio, no considero que sea la única ni la mejor, sino que es una alternativa coherente que permite abordar varias problemáticas, y sobre todo, permite dar cuenta de la responsabilidad del arqueólogo al interpretar el pasado. A partir de un enfoque semiótico, el abismo entre el 
pasado y el presente desaparece, ya que las interpretaciones de la cultura material se realizan en el presente con el objetivo de incidir sobre el presente (Funari et al. 2005; Shanks y Tilley 1992).

La combinación entre la Teoría de la Práctica, la semiótica y la performance resulta una alternativa viable porque permite aplicar una perspectiva uniforme a las relaciones entre los sujetos y objetos en el tiempo y el espacio. Y este punto es fundamental a la hora de relacionar las interpretaciones del pasado con el presente. Además, como mencioné anteriormente, permite abordar una serie de problemáticas de la materialidad con un enfoque novedoso (por ejemplo, la manera en que la materialidad construye relaciones temporales, ver Vaquer en prensa).

Como conclusión, la manera de evaluar si el presente enfoque es rentable es aplicarlo a más casos y situaciones específicas. Más allá de compartir o no la propuesta, la considero válida por el hecho de que nos permite replantearnos nuestra responsabilidad como investigadores. Y la virtud de cualquier marco no es generar respuestas, sino permitir plantearnos más y mejores preguntas...

Agradecimientos

Quiero agradecer a Axel Nielsen por introducirme en la semiótica de Peirce, y remarcar la relación entre Arqueología Conductual y materialidad. A los dos evaluadores anónimos por los comentarios que ayudaron a enriquecer el trabajo. El producto final es de mi entera responsabilidad.

\section{Bibliografía Citada}

Barnes, B.

2001 The Macro / Micro Problem and the Problem of Structure and Agency. Handbook of Social Theory (ed. por G. Ritzer y B. Smart), pp. 339-352. Sage Publications, Londres.

Barrett, J. C.

1994 Fragments of Antiquity. An Archaeology of Social Life in Britain, 2900 - 1200 BC. Indo American Books, Delhi.

Binford, L. R.

1994 En busca del pasado. Crítica, Barcelona.

1999 Willow Smoke and Dog's Tails: Hunter - Gatherer Settlement Systems and Archaeological Site Formation. Contemporary Archaeology in Theory. A Reader (ed. por R. Preucel e I. Hodder), pp. 39-60. Blackwell Publishers, Oxford.

Bourdieu, P.

1977 Outline of a Theory of Practice. Cambridge University Press, Cambridge. 1990 The Logic of Practice. Routledge, Londres.

2002 Razones Prácticas. Sobre la Teoría de la Acción. Anagrama. Barcelona.

Callinicos, A.

2004 Making History. Agency, Structure and Change in Social Theory. Brill, Leiden y Boston.

Crook, S.

2001 Social Theory and the Postmodern. Handbook of Social Theory, (ed. por G. Ritzer y B. Smart), pp. 308-323. Sage Publications, Londres. 
Funari, P., A. Zarankin y E. Stovel

2005 Global Archaeological Theory. Introduction. Global Archaeological Theory. Contextual Voices and Contemporary Thoughts, (ed. por P. Funari, A. Zarankin y E. Stovel), pp. 1-10. Kluwer Academic / Plenum Press, Nueva York.

Giddens, A.

1998 La Constitución de la Sociedad. Bases para la Teoría de la Estructuración. Amorrortu Editores. Buenos Aires.

2001 Las Nuevas Reglas del Método Sociológico. Crítica Positiva de las Sociologías Comprensivas. Amorrortu Editores, Buenos Aires.

González - Ruibal, A.

2007 Arqueología Simétrica: un giro teórico sin revolución paradigmática. Complutum 18: 283-285.

Hodder, I.

1990 The Domestication of Europe. Structure and Contingency in Neolithic Societies. Basil Blackwell, Oxford.

2001 Introduction: A Review of Contemporary Theoretical Debates in Archaeology. Archaeological Theory Today (ed. por I. Hodder), pp. 1-13. Polity Press, Cambridge.

2007 The "Social" in Archaeological Theory. An Historical and Contemporary Perspective. A Companion to Social Archaeology (ed. por L. Meskell y R. Preucel), pp. 23-42. Blackwell Publishers, Oxford.

Jones, A.

2007 Memory and Material Culture. Cambridge University Press, Cambridge.

Keane, W.

2005 Signs Are Not the Garb of Meaning: On the Social Analysis of Material Things. Materiality (ed. por D. Miller), pp. 182-205. Duke University Press, Durham.

2007 Christian Moderns. Freedom and Fetish in the Mission Encounter. University of California Press, Berkeley, Los Angeles y Londres

LaMotta, V. y Schiffer, M. B.

2001Behavioral Archaeology. Toward a New Synthesis. Archaeological Theory Today (ed. por I. Hodder), pp. 14- 64. Polity Press, Cambridge.

Latour, B.

2005 Reassembling the Social. An Introduction to Actor - Network - Theory. Oxford University Press, Oxford.

Lazzari, M.

2005 The Texture of Things: Objects, People and Landscape in Northwestern Argentina (First Millenium AD). Archaeologies of Materiality (ed. por L. Meskell), pp. 126 - 161. Blackwell Publishing. Oxford.

Leonard, R. 2001 Evolutionary Archaeology. Archaeological Theory Today (ed. por I. Hodder), pp. 65-97. Polity Press, Cambridge. 
Meskell, L.

2004 Objects Worlds in Ancient Egypt. Material Biographies Past and Present. Berg, Londres y Nueva York.

Miller, D.

2005 Materiality. An Introduction. Materiality (ed. por D. Miller), pp. 1-50. Duke University Press, Durham.

Nielsen, A. E.

1995 Architectural Performance and the Reproduction of Social Power. Expanding Archaeology (ed. por J. Skibo, W. Walker y A. Nielsen), pp. 47-66. University of Utah Press, Salt Lake City.

2007a Armas significantes: tramas culturales, guerra y cambio social en el Sur Andino prehispánico. Boletín del Museo Chileno de Arte Precolombino Vol. 12, No. 1: 4- 41.

2007b Bajo el hechizo de los emblemas: políticas corporativas y tráfico interregional en los Andes Circumpuneños. Producción y circulación prehispánicas de bienes en el Sur Andino (comp. por A. Nielsen, M. Rivolta, V. Seldes, M. Vázquez y P. Mercolli), pp. 393- 412. Editorial Brujas, Córdoba.

Olsen, B.

2003 Material Culture after Text: Re - Membering Things. Norwegian Archaeological Review 36 (2): 87-104.

Parker Pearson, M. y C. Richards 1994. Ordering the world: perceptions of architecture, space and time. Architecture and Order. Approaches to Social Space (ed. por M. Parker Pearson y C. Richards), pp. 1-37. Routledge, Londres.

Pauketat, T.

2001a Practice and History in Archaeology: An Emerging Paradigm. Anthropological Theory 1 (1): 73-98.

2001b. The Archaeology of Traditions. Agency and History Before and After Columbus. University of Florida Press, Gainesville.

Preucel, R.

2006 Archaeological Semiotics. Blackwell, Oxford.

Politis, G. y Pérez Gollán, J. A.

2007 Latin American Archaeology: from Colonialism to Globalization. A Companion to Social Archaeology (ed. por L. Meskell y R. Preucel), pp. 353-373. Blackwell Publishers, Oxford.

Renfrew, C.

1994 Towards a Cognitive Archaeology. The Ancient Mind. Elements of Cognitive Archaeology (ed. por C. Renfrew y E. Zubrow), pp. 3-12. Cambridge University Press, Cambridge.

Ritzer, G. y D. Goodman

2001 Postmodern Social Theory. Handbook of Sociological Theory (ed. por J. Turner), pp. 151170. Springer, Los Ángeles. 
Ritzer, G. y B. Smart

2001 Introduction: Theorists, Theories and Theorizing. Handbook of Social Theory (ed. por G. Ritzer y B. Smart), pp. 1-9. Sage Publications, Londres.

Salatino, P.

2008 Imágenes sobre las rocas: construcción del paisaje social en Chile central. Análisis espacial de sitios con petroglifos del cerro Tuququre, Valle del Putaendo, Región Aconcagua. Tesis de Licenciatura, Universidad de Buenos Aires, Facultad de Filosofía y Letras, Buenos Aires.

Saussure, F.

1993 [1916]. Curso de Lingüística General. Planeta - Agostini, Buenos Aires.

Schiffer, M. B.

1999 The Material Life of Human Beings. Routledge, Londres.

Shanks, M. y C. Tilley

1992 Re - Constructing Archaeology. Theory and Practice. Segunda Edición. Routledge, Londres.

Shanks, M. e I. Hodder

1995 Processual, postprocessual and interpretative archaeologies. Interpreting archaeology. Finding meaning in the past (ed. por I. Hodder, M. Shanks, A. Alexandri, V. Buchli, J. Carman, J. Last y G. Lucas), pp. 3-29. Routledge, Londres y Nueva York.

Short, $\mathrm{T}$.

2007 Peirce's Theory of Signs. Cambridge University Press, Nueva York.

Thomas, J.

1996 Time, Culture and Identity. An interpretive archaeology. Routledge, Londres y Nueva York.

2005 Materiality and the Social. Global Archaeological Theory. Contextual Voices and Contemporary Thoughts, (ed. por P. Funari, A. Zarankin y E. Stovel), pp. 11-18. Kluwer Academic / Plenum Press, Nueva York.

Tilley, C.

1994 A Phenomenology of Landscape. Places, Paths and Monuments. Berg, Oxford.

2006 Objectification. Handbook of Material Culture (ed. por C. Tilley, W. Keane, S. Küchler, M. Rowlands y P. Spyer), pp. 60- 73. Sage Publications, Londres.

Vaquer, J. M.

2010a Personas corporativas, sociedades corporativas: conflicto, prácticas sociales e incorporación en Cruz Vinto (Norte de Lípez, Potosí, Bolivia) durante el Periodo de Desarrollos Regionales Tardío (1200 - 1450 DC). Intersecciones en Antropología 11: 199- 213.

2010b Arquitectura, performance y percepción en Cruz Vinto (Norte de Lípez, Bolivia). Un ensayo metodológico. Arqueología Argentina en el Bicentenario de la Revolución de Mayo. Trabajos del XVII Congreso Nacional de Arqueología Argentina. Tomo II (ed. por J. Bárcena y H. Chiavazza), pp. 461-466. Universidad Nacional de Cuyo y ANPCyT, Mendoza.

2011a Paisaje, materialidad y prácticas sociales en Cruz Vinto. Editorial Académica Española, 
Saarbrücken.

2011b El Tiempo de los Ancestros: Temporalidad, Ideología Semiótica y Poder en Cruz Vinto (Norte de Lípez, Bolivia) durante el Periodo de Desarrollos Regionales Tardío (1200 - 1450 DC). Revista de Arqueología Sudamericana. En prensa.

Vaquer, J. M., E. A. Calomino y V. N. Zuccarelli

2010 Habitando Cruz Vinto: Temporalidad y Espacialidad en un pukara del Periodo de Desarrollos Regionales Tardío (1200 - 1450 DC) en el Norte de Lípez (Potosí, Bolivia). Arqueología 16: 13- 33. 